\title{
PINNED-FLAGS OF SOME OPERATIONS ON FUZZY SUBGROUPS
}

\author{
B. B. MAKAMBA AND V. MURALI
}

Received 22 September 2004 and in revised form 15 September 2005

Fuzzy subgroups of finite groups have been treated recently using the concept of pinnedflags. In this paper, we consider the operations of intersection, sum, product, and quotient of fuzzy subgroups of finite abelian groups in general, in terms of pinned-flags. We develop algorithms to construct pinned-flags of fuzzy subgroups corresponding to these operations and prove their validity. We illustrate some applications of such algorithms.

\section{Introduction}

Some of the past studies of fuzzy subgroups relied heavily on the usual definitions of intersection, union, sum of two fuzzy subgroups by exploiting the lattice properties of membership values in a simplistic way. For instance, the intersection of two fuzzy subgroups $\mu$ and $\nu$ is given by $(\mu \wedge \nu)(g)=\mu(g) \wedge \nu(g)$; the sum is given by $(\mu+\nu)(g)=\bigvee_{g_{1} g_{2}=g}\left(\mu\left(g_{1}\right)\right.$ $\left.\wedge \nu\left(g_{2}\right)\right)$, for $g \in G$, and so forth. On the other hand, one form of representation of fuzzy subgroups, that proved useful in the literature, is by means of their $\alpha$-cuts. By refining the chain formed by the $\alpha$-cuts, we get a maximal chain of subgroups which is called a flag. The membership values of elements form a decreasing chain of real numbers in the unit interval known as keychain. A pinned-flag is a pair consisting of a flag and a keychain. One can study the operations on fuzzy subgroups by means of pinned-flags, thus enriching some properties of fuzzy subgroups. It was observed in [6] that the pinnedflag resulting from operations of intersection and direct sum of fuzzy subgroups does not form any particular pattern. Example 4.6 of the same paper [6] illustrated the peculiarity of the determination of pinned-flags for $\mu \wedge \nu$ and $\mu+\nu$ in terms of the pinned-flags of $\mu$ and $\nu$. As a consequence, the complete characterization of these operations in terms of pinned-flags was left open in that paper. We develop algorithms to describe the pinnedflags of the intersection, sum, product, and quotient of fuzzy subgroups and prove their validity.

In Section 2, we gather all the preliminaries such as flags, chains and keychains, pinned-flags of fuzzy subgroups and fix notations. In Section 3, given pinned-flags of two fuzzy subgroups, we develop algorithms to characterize the pinned-flags of their intersection and of their sum. In Section 4, we look at the operations of product and quotient as in Section 3. 


\section{Preliminaries}

We use $\mathbf{I}=[\mathbf{0}, \mathbf{1}]$, the real unit interval as a chain with the usual ordering in which $\wedge$ stands for infimum (inf) (or intersection) and $\vee$ stands for supremum (sup) (or union). Throughout this paper, we take $G$ to be a finite abelian group of order $N$ and take $G_{0}$ to be the trivial subgroup $\{0\}$. Even though almost all results of this paper are applicable to any finite group, abelian or not, we use + for the group operation and 0 for the identity element for convenience of notation, rather than the more conventional multiplicative notation. By an $\alpha$-cut of $\mu$, for a real number $\alpha$ in $\mathbf{I}$, we mean a subset $\mu^{\alpha}=\{x \in G: \mu(x) \geq$ $\alpha$ \} of $G$. A fuzzy set $\mu$ is said to be a fuzzy subgroup if $\mu(x+y) \geq \mu(x) \wedge \mu(y)$ for all $x, y \in G$ and $\mu(x)=\mu(-x)$, see [7]. We assume that $\mu(0)=1$ throughout this paper. For any fuzzy subgroup $\mu$ of $G$, the $\alpha$-cut $\mu^{\alpha}$ is a subgroup of $G$. By core and support of $\mu$, we mean the crisp subsets of $G$ given by $\{x \in G: \mu(x)=1\}$ and $\{x \in G: \mu(x) \neq 0\}$, respectively. For later use, we recall the definitions of sum and product of two fuzzy subgroups $\mu$ and $\nu$,

$$
\begin{gathered}
(\mu+\nu)(x)=\sup \left\{\mu\left(x_{1}\right) \wedge \nu\left(x_{2}\right): x_{1}+x_{2}=x ; x, x_{1}, x_{2} \in G\right\}, \\
(\mu \times \nu)(x, y)=\mu(x) \wedge \nu(y),
\end{gathered}
$$

respectively. For results on product, see $[2,8]$. We refer the reader to [6] for results on flag, keychain, and pinned-flag, but state their definitions here. By a flag $\mathscr{C}$ on $G$, we mean a maximal chain of subgroups of the form

$$
\{0\}=G_{0} \subset G_{1} \subset G_{2} \subset \cdots \subset G_{n}=G .
$$

We call various $G_{i}$ 's the components of the flag $\mathscr{C}$; in particular, $G_{i}$ is called the $i$ th component of the flag. From the Jordan-Hölder theorem, it is clear that any two flags of $G$ are of the same length. We assume this length to be $n+1$ for some fixed $n$ less than $N$. form

By a keychain $\ell$, we mean an $(n+1)$-tuple $\left(\lambda_{0}, \lambda_{1}, \ldots, \lambda_{n}\right)$ of real numbers in $\mathbf{I}$ of the

$$
1=\lambda_{0} \geq \lambda_{1} \geq \lambda_{2} \geq \cdots \geq \lambda_{n} \geq 0
$$

The $\lambda_{i}$ 's are called pins. The length of keychain $\ell$ is $n+1$.

Definition 2.1. A pinned-flag on $G$ is a pair $(\mathscr{C}, \ell)$ consisting of a falg $\mathscr{C}$ on $G$ and a keychain $\ell$ from $I$, written as follows:

$$
G_{0}^{1} \subset G_{1}^{\lambda_{1}} \subset G_{2}^{\lambda_{2}} \subset \cdots \subset G_{n}^{\lambda_{n}}
$$

$G_{i}^{\lambda_{i}}$, for $i=0,1, \ldots, n$, is called the $i$ th component of the pinned-flag. With the pinned-flag $(\mathscr{b}, \ell)$, a fuzzy subgroup

$$
\mu=\bigvee_{i=0}^{n} \lambda_{i} \chi_{\mu^{\lambda_{i}}}
$$

can be associated. It is easily checked that $\mu$ as defined above is a fuzzy subgroup of $G$. 
Conversely, a pinned-flag $\left(\mathscr{C}_{\mu}, \ell_{\mu}\right)$ is associated with a given fuzzy subgroup $\mu$ by refining the $\alpha$-cuts to yield a flag $\mathscr{C}_{\mu}$ on $G$ and by repeating the membership values if necessary to yield the corresponding keychain $\ell_{\mu}$. The following example illustrates the process.

Example 2.2. Consider a fuzzy subgroup $\mu: \mathbb{Z}_{72} \rightarrow \mathbf{I}$ defined by

$$
\mu(x)= \begin{cases}1, & x \in \mathbb{Z}_{3}, \\ \lambda_{1}, & x \in \mathbb{Z}_{18} \backslash \mathbb{Z}_{3}, \\ \lambda_{2}, & x \in \mathbb{Z}_{36} \backslash \mathbb{Z}_{18}, \\ \lambda_{3}, & x \in \mathbb{Z}_{72} \backslash \mathbb{Z}_{36} .\end{cases}
$$

The chain of subgroups obtained by $\alpha$-cuts of $\mu$ can be refined to a flag $\{0\} \subset \mathbb{Z}_{3} \subset$ $\mathbb{Z}_{9} \subset \mathbb{Z}_{18} \subset \mathbb{Z}_{36} \subset \mathbb{Z}_{72}$. The corresponding pinned-flag is $\left(\mathscr{C}_{\mu}, \ell_{\mu}\right):\{0\}^{1} \subset \mathbb{Z}_{3}^{1} \subset \mathbb{Z}_{9}^{\lambda_{1}} \subset \mathbb{Z}_{18}^{\lambda_{1}} \subset$ $\mathbb{Z}_{36}^{\lambda_{2}} \subset \mathbb{Z}_{72}^{\lambda_{3}}$ and its keychain is $\ell_{\mu}:\left(1,1, \lambda_{1}, \lambda_{1}, \lambda_{2}, \lambda_{3}\right)$. Since the $\alpha$-cuts did not form a flag, the refinement of the $\alpha$-cuts to a flag has given rise to repetition of some pins in the keychain $\ell_{\mu}$.

\section{Pinned-flags for intersection and sum of fuzzy subgroups}

In this section, we give algorithms to find the pinned-flags for intersection and the sum of two fuzzy subgroups whose pinned-flags are known. Suppose the pinned-flags corresponding to two fuzzy subgroups $\mu$ and $\nu$ of $G$ are given by

$$
\begin{gathered}
\left(\mathscr{C}_{\mu}, \ell_{\mu}\right): G_{0}^{1} \subset G_{1}^{\lambda_{1}} \subset \cdots \subset G_{n}^{\lambda_{n}}, \\
\left(\mathscr{C}_{\nu}, \ell_{\nu}\right): H_{0}^{1} \subset H_{1}^{\beta_{1}} \subset \cdots \subset H_{n}^{\beta_{n}} . \quad \text { Then } \\
\left(\mathscr{C}_{\mu \wedge \nu}, \ell_{\mu \wedge \nu}\right): K_{0}^{1} \subset K_{1}^{\gamma_{1}} \subset \cdots \subset K_{n}^{\gamma_{n}}
\end{gathered}
$$

is the pinned-flag for $\mu \wedge \nu$.

Algorithm 3.1

Step 1. Firstly, $K_{0}^{1}=G_{0}^{1}=H_{0}^{1}$. We usually denote this by simply $0^{1}$ in all cases.

Step 2. Find the least $j$ and $i$ such that $G_{1} \subseteq H_{j}$ for $1 \leq j \leq n$ and $H_{1} \subseteq G_{i}$ for $1 \leq i \leq n$, respectively. Then $\gamma_{1}=\left(\lambda_{1} \wedge \beta_{j}\right) \vee\left(\lambda_{i} \wedge \beta_{1}\right)$ and

$$
\text { we define } K_{1}= \begin{cases}G_{1} & \text { if } \gamma_{1}=\lambda_{1} \wedge \beta_{j} \\ H_{1} & \text { if } \gamma_{1}=\lambda_{i} \wedge \beta_{1}\end{cases}
$$

Step 3 (inductive step). Suppose we have defined $K_{s}^{\gamma_{s}}$ with either $K_{s}=G_{s}$ or $K_{s}=H_{s}$. To obtain $K_{s+1}^{\gamma_{s+1}}$, we proceed as follows: find the least indices $j$ and $i$ such that $G_{s+1} \subseteq H_{j}$ for $s+1 \leq j \leq n$, and similarly $H_{s+1} \subseteq G_{i}$ for $s+1 \leq i \leq n$, respectively. 
Case 1. $K_{s} \subseteq G_{s+1} \cap H_{s+1}$. Then $\gamma_{s+1}=\left(\lambda_{s+1} \wedge \beta_{j}\right) \vee\left(\lambda_{i} \wedge \beta_{s+1}\right)$ and

$$
K_{s+1}= \begin{cases}G_{s+1} & \text { if } \gamma_{s+1}=\lambda_{s+1} \wedge \beta_{j} \\ H_{s+1} & \text { if } \gamma_{s+1}=\lambda_{i} \wedge \beta_{s+1}\end{cases}
$$

Case 2. $K_{s} \nsubseteq G_{s+1} \cap H_{s+1}$. Then either $K_{s} \subset G_{s+1}$ or $K_{s} \subset H_{s+1}$ only. In these cases, $\gamma_{s+1}$ is defined, respectively, as $\lambda_{s+1} \wedge \beta_{j}$ or $\lambda_{i} \wedge \beta_{s+1}$. If $K_{s}=K_{n}$, the algorithm terminates.

Next, we illustrate the above procedure with an example (the same example as in $[6$, Example 4.6]).

Example 3.2. Consider two fuzzy subgroups $\mu$ and $\nu$ of $G=\mathbb{Z}_{72}$, a cyclic group of order 72 with the following pinned-flags:

$$
\begin{aligned}
& \left(\mathscr{C}_{\mu}, \ell_{\mu}\right): G_{0}^{1} \subset \mathbb{Z}_{3}^{1 / 2} \subset \mathbb{Z}_{9}^{1 / 5} \subset \mathbb{Z}_{18}^{1 / 6} \subset \mathbb{Z}_{36}^{1 / 9} \subset \mathbb{Z}_{72}^{1 / 10}, \\
& \left(\mathscr{C}_{\nu}, \ell_{\nu}\right): H_{0}^{1} \subset \mathbb{Z}_{3}^{1 / 3} \subset \mathbb{Z}_{6}^{1 / 4} \subset \mathbb{Z}_{12}^{1 / 7} \subset \mathbb{Z}_{36}^{1 / 8} \subset \mathbb{Z}_{72}^{1 / 11} .
\end{aligned}
$$

Carrying out Algorithm 3.1, we get the following pinned-flag for the intersection of $\mu$ and $v$ :

$$
\left(\mathscr{C}_{\mu \wedge \nu}, \ell_{\mu \wedge \nu}\right): 0^{1} \subset \mathbb{Z}_{3}^{1 / 3} \subset \mathbb{Z}_{6}^{1 / 6} \subset \mathbb{Z}_{18}^{1 / 8} \subset \mathbb{Z}_{36}^{1 / 9} \subset \mathbb{Z}_{72}^{1 / 11}
$$

Note 3.3. In this note, we justify that the above algorithm indeed yields the pinned-flag corresponding to the intersection $\mu \wedge \nu$. Therefore, let $x \in G$ and find the least subscripts $i$ and $j$ such that $x \in G_{i} \backslash G_{i-1}$ and $x \in H_{j} \backslash H_{j-1}$ so that $(\mu \wedge \nu)(x)=\lambda_{i} \wedge \beta_{j}$. We must show now that if $x \in K_{s} \backslash K_{s-1}$, then $\gamma_{s}=\lambda_{i} \wedge \beta_{j}$.

Now, either $K_{s}=G_{s}$ or $K_{s}=H_{s}$. Suppose that $K_{s}=G_{s}$, then $s=i$; for otherwise $s>i$, implying that $K_{s} \subset K_{i}$. But $K_{i}$ is either $G_{i}$ or $H_{i}$ by the algorithm. This leads to a contradiction. Therefore, $K_{s}=K_{i}=G_{i}$ or $K_{s}=K_{j}=H_{j}$. From the algorithm again, $\gamma_{s}=\lambda_{i} \wedge \beta_{j}$.

We next construct the pinned-flag of the sum $\mu+\nu$ of two fuzzy subgroups from their associated pinned-flags. Suppose that $\mu$ and $\nu$ are represented by pinned-flags as in (3.1). Algorithm 3.4 constructs the pinned-flag $\left(\mathscr{C}_{\mu+\nu}, \ell_{\mu+\nu}\right): K_{0}^{1} \subset K_{1}^{\gamma_{1}} \subset \cdots \subset K_{n}^{\gamma_{n}}$ for $\mu+\nu$.

\section{Algorithm 3.4}

Step 1. Firstly, $K_{0}^{1}=G_{0}^{1}=H_{0}^{1}$. We usually denote this by simply $0^{1}$ in all cases.

Step 2. To find $K_{1}^{\gamma_{1}}$, the second component of the pinned-flag, we proceed as follows: let $\gamma_{1}=\lambda_{1} \vee \beta_{1}$. Then $K_{1}=G_{1}$ if $\gamma_{1}=\lambda_{1}$. Otherwise, $K_{1}=H_{1}$.

Step 3 (inductive step). Suppose that we have defined $K_{s}^{\gamma_{s}}$. To obtain $K_{s+1}^{\gamma_{s+1}}$, we proceed as follows: we base our cases on whether both $G_{s+1}$ and $H_{s+1}$ split or not.

Case 1. Suppose that both do not split, then set $\gamma_{s+1}=\lambda_{s+1} \vee \beta_{s+1}$ and $K_{s+1}=G_{s+1}$ if $\gamma_{s+1}=\lambda_{s+1}$, otherwise $K_{s+1}=H_{s+1}$. 
Case 2. Suppose that one of them splits and the other does not, say, $G_{s+1}=G_{i}+H_{j}$ for some indices $i$ and $j$ (choose the least values), then set $\gamma_{s+1}=\left(\lambda_{i} \wedge \beta_{j}\right) \vee \lambda_{s+1} \vee \beta_{s+1}$ and $K_{s+1}=G_{s+1}$ when $\gamma_{s+1}=\left(\lambda_{i} \wedge \beta_{j}\right) \vee \lambda_{s+1}$, otherwise $K_{s+1}=H_{s+1}$ provided that $K_{s} \subseteq G_{s+1} \cap$ $H_{s+1}$. If the last condition is not satisfied, then simply $K_{s+1}^{\gamma_{s+1}}=G_{s+1}^{\left(\lambda_{i} \wedge \beta_{j}\right) \vee \lambda_{s+1}}$ provided that $K_{s} \subseteq G_{s+1}$ and $K_{s} \nsubseteq H_{s+1}$. We handle the case when $G$ and $H$ swop places similarly.

Case 3. When both $G_{s+1}$ and $H_{s+1}$ split, firstly they are equal. Secondly, we follow the steps in Case 2. If $K_{s}=K_{n}$, the algorithm terminates.

Carrying out the above algorithm in Example 3.2, we get the following pinned-flag for the $\operatorname{sum} \mu+v$ :

$$
\left(\mathscr{C}_{\mu+\nu}, \ell_{\mu+\nu}\right): 0^{1} \subset \mathbb{Z}_{3}^{1 / 2} \subset \mathbb{Z}_{6}^{1 / 4} \subset \mathbb{Z}_{18}^{1 / 5} \subset \mathbb{Z}_{36}^{1 / 7} \subset \mathbb{Z}_{72}^{1 / 10}
$$

Note 3.5. The argument for the justification of the above algorithm is similar to the one given in Note 3.3, and therefore omitted here.

\section{Pinned-flags for product and quotient of fuzzy subgroups}

In this section, we study the operations of product and quotient. We describe below algorithms to find the pinned-flags for the product and quotient of two fuzzy subgroups. First, we take up the product operation.

Suppose that the pinned-flags corresponding to two fuzzy subgroups $\mu$ of $G$ and $\nu$ of $H$ are given by

$$
\begin{aligned}
& \left(\mathscr{C}_{\mu}, \ell_{\mu}\right): G_{0}^{1} \subset G_{1}^{\lambda_{1}} \subset \cdots \subset G_{n}^{\lambda_{n}}, \\
& \left(\mathscr{C}_{\nu}, \ell_{\nu}\right): H_{0}^{1} \subset H_{1}^{\beta_{1}} \subset \cdots \subset H_{m}^{\beta_{m}} .
\end{aligned}
$$

The pinned-flag $\left(\mathscr{C}_{\mu \times v}, \ell_{\mu \times v}\right): K_{0}^{1} \subset K_{1}^{\gamma_{1}} \subset \cdots \subset K_{p}^{\gamma_{p}}$ for $\mu \times \nu$ is constructed as follows. Algorithm 4.1

Step 1. Firstly, $K_{0}^{1}=\left(G_{0} \times H_{0}\right)^{1}$. We usually denote this by simply $(0 \times 0)^{1}$ in all cases. Step 2. To find $K_{1}^{\gamma_{1}}$, the second component of the pinned-flag, we proceed as follows:

$$
\text { Let } \gamma_{1}=\beta_{1} \vee \lambda_{1}, \quad \text { then } K_{1}= \begin{cases}G_{1} \times H_{0} & \text { if } \gamma_{1}=\lambda_{1} \text {, } \\ G_{0} \times H_{1} & \text { if } \gamma_{1}=\beta_{1} .\end{cases}
$$

Step 3 (inductive step). Suppose that we have defined $K_{s-1}^{\gamma_{s-1}}$. To obtain $K_{s}^{\gamma_{s}}$, we proceed as follows: suppose that $K_{s-1} \subseteq\left(G_{s} \times H_{0}\right) \cap\left(G_{s-1} \times H_{1}\right) \cap\left(G_{s-2} \times H_{2}\right) \cap \cdots \cap\left(G_{0} \times H_{s}\right)$. 
Let $\gamma_{s}=\left(\lambda_{0} \wedge \beta_{s}\right) \vee\left(\lambda_{1} \wedge \beta_{s-1}\right) \vee\left(\lambda_{2} \wedge \beta_{s-2}\right) \vee\left(\lambda_{3} \wedge \beta_{s-3}\right) \vee \cdots \vee\left(\lambda_{s-1} \wedge \beta_{1}\right) \vee\left(\lambda_{s} \wedge \beta_{0}\right)$.

$$
\text { Then } K_{s}=\left\{\begin{array}{cl}
G_{s} \times H_{0} & \text { if } \gamma_{s}=\lambda_{s}, \\
G_{s-1} \times H_{1} & \text { if } \gamma_{s}=\lambda_{s-1} \wedge \beta_{1}, \\
G_{s-2} \times H_{2} & \text { if } \gamma_{s}=\lambda_{s-2} \wedge \beta_{2}, \\
\vdots & \\
G_{1} \times H_{s-1} & \text { if } \gamma_{s}=\lambda_{1} \wedge \beta_{s-1}, \\
G_{0} \times H_{s} & \text { if } \gamma_{s}=\beta_{s} .
\end{array}\right.
$$

Suppose for some indices $i$ and $j$ that the products $G_{i} \times H_{j},(i+j=s)$ do not contain $K_{s-1}$, then

(i) $\gamma_{s}$ is defined as above except that the pins $\lambda_{i} \wedge \beta_{j}$ of the $G_{i} \times H_{j}$ are excluded in taking the supremum,

(ii) $K_{s}$ is defined as above except that the $G_{i} \times H_{j}$ are excluded from the list of possibilities for $K_{s}$.

When $s=p=n+m$ the algorithm terminates. It is clear that the chain formed by $K_{s}$ 's is maximal and the number of components is $p+1=n+m+1$.

We illustrate Algorithm 4.1 with an example below.

Example 4.2. Consider fuzzy subgroups $\mu$ and $\nu$ of the groups $G=\mathbb{Z}_{63}$ and $H=\mathbb{Z}_{40}$, respectively, given by the pinned-flags

$$
\begin{aligned}
& \left(\mathscr{C}_{\mu}, \ell_{\mu}\right): 0^{1} \subset \mathbb{Z}_{3}^{1 / 2} \subset \mathbb{Z}_{21}^{1 / 3} \subset \mathbb{Z}_{63}^{1 / 5}, \\
& \left(\mathscr{C}_{\nu}, \ell_{\nu}\right): 0^{1} \subset \mathbb{Z}_{2}^{3 / 4} \subset \mathbb{Z}_{10}^{1 / 4} \subset \mathbb{Z}_{20}^{1 / 5} \subset \mathbb{Z}_{40}^{1 / 6} .
\end{aligned}
$$

The pinned-flag $\left(\mathscr{C}_{\mu \times \nu}, \ell_{\mu \times \nu}\right)$ for $\mu \times \nu$ in this example has 8 components including the first zero component, and is given by

$$
\begin{aligned}
(0 \times 0)^{1} & \subset\left(0 \times \mathbb{Z}_{2}\right)^{3 / 4} \subset\left(\mathbb{Z}_{3} \times \mathbb{Z}_{2}\right)^{1 / 2} \subset\left(\mathbb{Z}_{21} \times \mathbb{Z}_{2}\right)^{1 / 3} \subset\left(\mathbb{Z}_{21} \times \mathbb{Z}_{10}\right)^{1 / 4} \\
& \subset\left(\mathbb{Z}_{63} \times \mathbb{Z}_{10}\right)^{1 / 5} \subset\left(\mathbb{Z}_{63} \times \mathbb{Z}_{20}\right)^{1 / 5} \subset\left(\mathbb{Z}_{63} \times \mathbb{Z}_{40}\right)^{1 / 6} .
\end{aligned}
$$

We now take up the study of the pinned-flags for the quotients. There are several notions of quotients of fuzzy subgroups used in the literature, see $[1,3,4,5,9]$. We work with one that is used more often. Note that in the following, we find it convenient to revert to the multiplicative notation when writing cosets of subgroups, for simplicity and conciseness.

Definition 4.3. Suppose $\mu$ and $v$ are two fuzzy subgroups of $G$. Then the fuzzy quotient group $\mu / \nu$ is defined as a fuzzy subgroup of the quotient group $G / \operatorname{core}(\nu)$ given by $(\mu / \nu)(x \operatorname{core}(\nu))=\sup \{\mu(a): a, \operatorname{core}(\nu)=x \operatorname{core}(\nu), a \in G\}$, see [3].

Algorithm 4.4. Suppose the pinned-flags for $\mu$ and $\nu$ are given as in (4.1). Since $\mu$ and $\nu$ are fuzzy subgroups of the same group $G, m=n$. If the core $(\nu)=G$, then the quotient is $G / \operatorname{core}(\nu)=\{e\}$. In this case, the pinned-flag of the quotient is simply $(G / \operatorname{core}(\nu))^{1}$ with only one component. Assume that $\operatorname{core}(\nu) \neq G$. So the $\operatorname{core}(\nu)=H_{i}$ for some $0 \leq i<n$. 
Case 1. Let $H_{i}=G_{j}$. Then firstly $i=j$. If not, either $i>j$ or $i<j$. The case $i<j$ implies that the maximal chain

$$
G_{0} \subset G_{1} \subset \cdots \subset G_{j-1} \subset G_{j}=H_{i} \subset H_{i+1} \subset \cdots \subset H_{n}
$$

has length $n-i+1+j=n+1+(j-i)>n+1$, a contradiction. Similarly, the case $i>j$ leads to a contradiction. Secondly,

$$
H_{i} / H_{i}=G_{i} / H_{i} \subset G_{i+1} / H_{i} \subset \cdots \subset G_{n} / H_{i}
$$

is clearly a maximal chain in $G / H_{i}$, leading to a pinned-flag

$$
\left(G_{i} / H_{i}\right)^{1} \subset\left(G_{i+1} / H_{i}\right)^{\gamma_{i+1}} \subset \cdots \subset\left(G_{n} / H_{i}\right)^{\gamma_{n}}
$$

for the quotient $\mu / \nu$, where $\gamma_{k}=\lambda_{k}$ if $G_{k}$ has no nontrivial decomposition. Otherwise, let $l$ and $m$ be the least subscripts such that $G_{k}=G_{l}+H_{m}$, then $\gamma_{k}=\left(\lambda_{l} \wedge \beta_{m}\right) \vee \lambda_{k}$.

Case $2\left(H_{i} \neq G_{i}\right)$. Choose the least subscript $k$ such that $H_{i} \subset G_{k}$. Clearly, the weighted chain

$$
\left(G_{k+1} / H_{i}\right)^{\gamma_{k+1}} \subset\left(G_{k+2} / H_{i}\right)^{\gamma_{k+2}} \subset \cdots \subset\left(G_{n} / H_{i}\right)^{\gamma_{n}}
$$

is part of a pinned-flag for $\mu / \nu$, where the $\gamma_{j}$ are as defined in Case 1 . We now extend the above weighted chain to a full pinned-flag for $\mu / \nu$. Choose the largest subscript $l_{1}<k$ such that $G_{l_{1}}+H_{i}=G_{k}$. Then, find the largest subscript $l_{2}$ such that $\left(G_{l_{1}}+H_{i}\right) / H_{i} \supset$ $\left(G_{l_{2}}+H_{i}\right) / H_{i}$, with $l_{1}>l_{2}$. Inductively, continue to find the largest $l_{i}$ such that $\left(G_{l_{i}}+\right.$ $\left.H_{i}\right) / H_{i} \subset\left(G_{l_{i-1}}+H_{i}\right) / H_{i}, k>l_{1}>l_{2}>\cdots>l_{s}$, and $\left(G_{l_{1}}+H_{i}\right) / H_{i} \supset\left(G_{l_{2}}+H_{i}\right) / H_{i} \supset \cdots \supset$ $\left(G_{l_{s}}+H_{i}\right) / H_{i}=H_{i} / H_{i}$. Consequently, a pinned-flag for the quotient $\mu / \nu$ is given by

$$
\begin{aligned}
\left(H_{i} / H_{i}\right)^{1} & \subset\left(\left(G_{l_{s-1}}+H_{i}\right) / H_{i}\right)^{\gamma_{l_{s-1}}} \subset \cdots \subset\left(\left(G_{l_{1}}+H_{i}\right) / H_{i}\right)^{\gamma_{l_{1}}} \\
& \subset\left(G_{k+1} / H_{i}\right)^{\gamma_{k+1}} \subset\left(G_{k+2} / H_{i}\right)^{\gamma_{k+2}} \subset \cdots \subset\left(G_{n} / H_{i}\right)^{\gamma_{n}} .
\end{aligned}
$$

Note that the $\gamma_{i}$ are as defined in Case 1. We illustrate the above construction with an example below.

Example 4.5. Suppose $\mu$ and $\nu$ are two fuzzy subgroups of $\mathbb{Z}_{72}$ given by

$$
\begin{aligned}
& \left(\mathscr{C}_{\mu}, \ell_{\mu}\right): G_{0}^{1} \subset \mathbb{Z}_{3}^{1 / 2} \subset \mathbb{Z}_{9}^{1 / 5} \subset \mathbb{Z}_{18}^{1 / 6} \subset \mathbb{Z}_{36}^{1 / 9} \subset \mathbb{Z}_{72}^{1 / 10}, \\
& \left(\mathscr{C}_{\nu}, \ell_{\nu}\right): H_{0}^{1} \subset \mathbb{Z}_{2}^{1} \subset \mathbb{Z}_{6}^{1} \subset \mathbb{Z}_{12}^{1} \subset \mathbb{Z}_{36}^{1 / 11} \subset \mathbb{Z}_{72}^{1 / 12} .
\end{aligned}
$$

By following the steps developed above, we get the pinned-flag for $\mu / \nu$ in this example as

$$
\left(\mathscr{C}_{\mu / \nu}, \ell_{\mu / \nu}\right):\left(\mathbb{Z}_{12} / \mathbb{Z}_{12}\right)^{1} \subset\left(\mathbb{Z}_{36} / \mathbb{Z}_{12}\right)^{1 / 5} \subset\left(\mathbb{Z}_{72} / \mathbb{Z}_{12}\right)^{1 / 10}
$$

Notice that $\mathbb{Z}_{36} / \mathbb{Z}_{12}$ has a membership value of $1 / 5$ since $\mathbb{Z}_{36}$ splits up into $\mathbb{Z}_{9}+\mathbb{Z}_{12}$. 


\section{Concluding remarks}

It is clear from a close look at Algorithms 4.1 and 4.4 that their constructions were based directly on the definitions of various operations on fuzzy subsgroups. Therefore, a justification is not necessary, but it is self-evident that these algorithms really indeed yield the correct pinned-flags corresponding to the respective operations on fuzzy subgroups. In conclusion, we wish to emphasize that the study of operations on fuzzy subgroups through their pinned-flags is important for at least two reasons. Firstly, they reflect the membership values of elements to the associated fuzzy subgroups in a transparent and useful way. Secondly, they bring out the essential features of the ways in which the membership values of elements to the constituent fuzzy subgroups are tied up to the membership values of the combined fuzzy subgroups under various operations.

\section{Acknowledgments}

The first author thanks The Govan Mbeki Research and Development Centre (GMRDC) of the University of Fort Hare, the second author thanks the Joint Research Committee (JRC) of Rhodes University, and both thank the National Research Foundation (NRF) of South Africa for support.

\section{References}

[1] P. Bhattacharya and N. P. Mukherjee, Fuzzy groups: some group theoretic analogs. II, Inform. Sci. 41 (1987), no. 1, 77-91.

[2] B. B. Makamba, Direct products and isomorphism of fuzzy subgroups, Inform. Sci. 65 (1992), no. $1-2,33-43$.

[3] M. Mashinchi and M. Mukaidono, Generalized fuzzy quotient subgroups, Fuzzy Sets and Systems 74 (1995), no. 2, 245-257.

[4] J. N. Mordeson, L-Subspaces and L-Subfields, Lecture Notes in Fuzzy Mathematics and Computer Science, Creighton University, Center for Research in Fuzzy Mathematics and Computer Science, Nebraska, 1996.

[5] N. P. Mukherjee and P. Bhattacharya, Fuzzy normal subgroups and fuzzy cosets, Inform. Sci. 34 (1984), no. 3, 225-239.

[6] V. Murali and B. B. Makamba, On an equivalence of fuzzy subgroups. III, Int. J. Math. Math. Sci. 2003 (2003), no. 36, 2303-2313.

[7] A. Rosenfeld, Fuzzy groups, J. Math. Anal. Appl. 35 (1971), no. 3, 512-517.

[8] H. Sherwood, Products of fuzzy subgroups, Fuzzy Sets and Systems 11 (1983), no. 1, 79-89.

[9] J. Zhou, S.-Y. Li, and S.-Y. Li, LP-fuzzy normal subgroups and fuzzy quotient groups, J. Fuzzy Math. 5 (1997), no. 1, 27-40.

[10] H.-J. Zimmermann, Fuzzy Set Theory-and Its Applications, International Series in Management Science/Operations Research, Kluwer-Nijhoff, Massachusetts, 1985.

B. B. Makamba: Department of Mathematics (Pure and Applied), University of Fort Hare, Alice 5700, South Africa

E-mail address: bmakamba@ufh.ac.za

V. Murali: Department of Mathematics (Pure and Applied), Rhodes University, Grahamstown 6140, South Africa

E-mail address: v.murali@ru.ac.za 


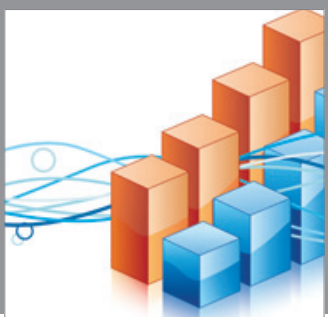

Advances in

Operations Research

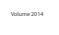

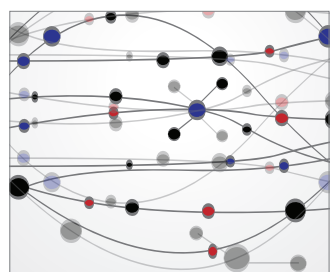

\section{The Scientific} World Journal
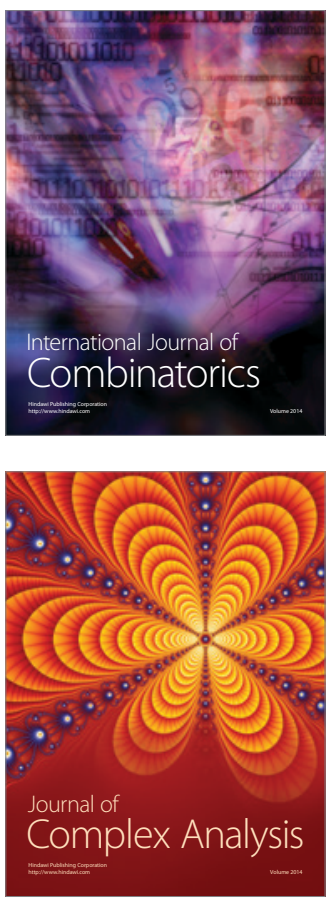

International Journal of

Mathematics and

Mathematical

Sciences
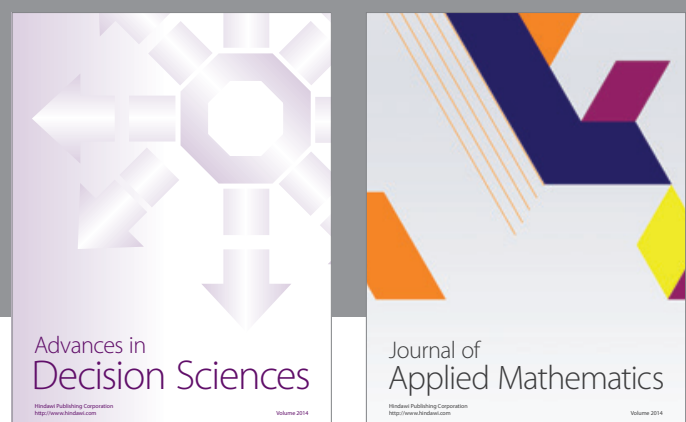

Journal of

Applied Mathematics
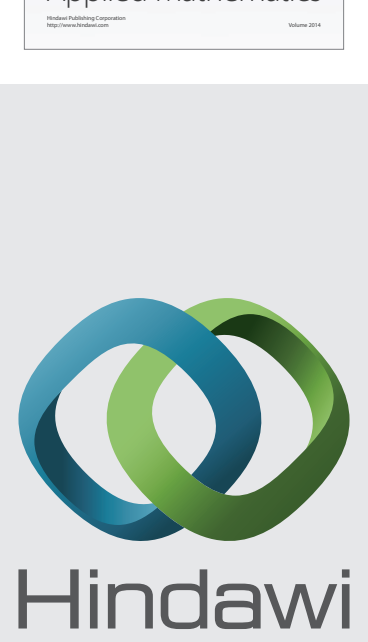

Submit your manuscripts at http://www.hindawi.com
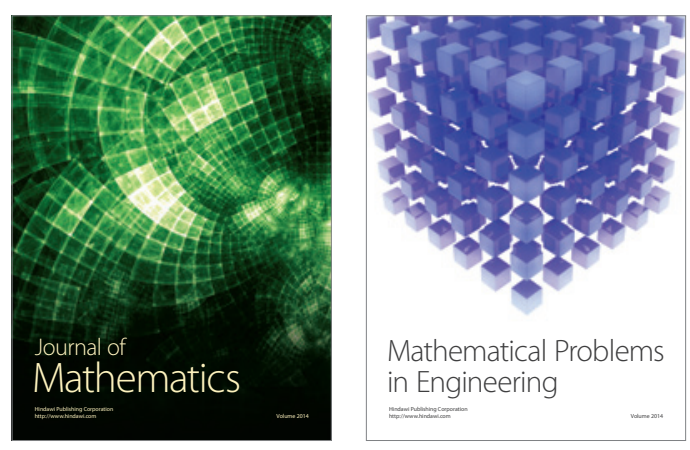

Mathematical Problems in Engineering
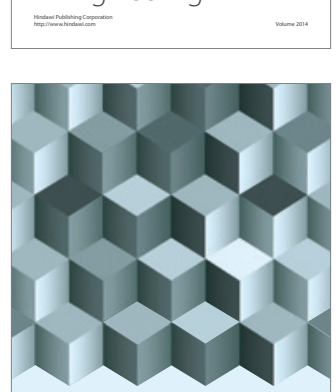

Journal of

Function Spaces
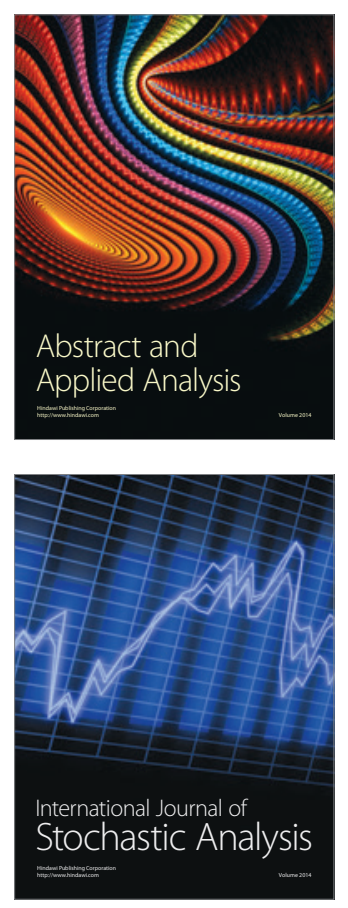

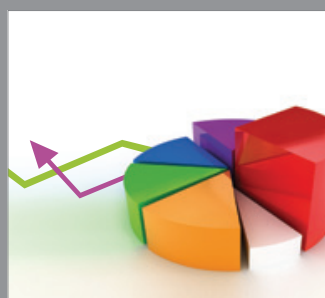

ournal of

Probability and Statistics

Promensencen
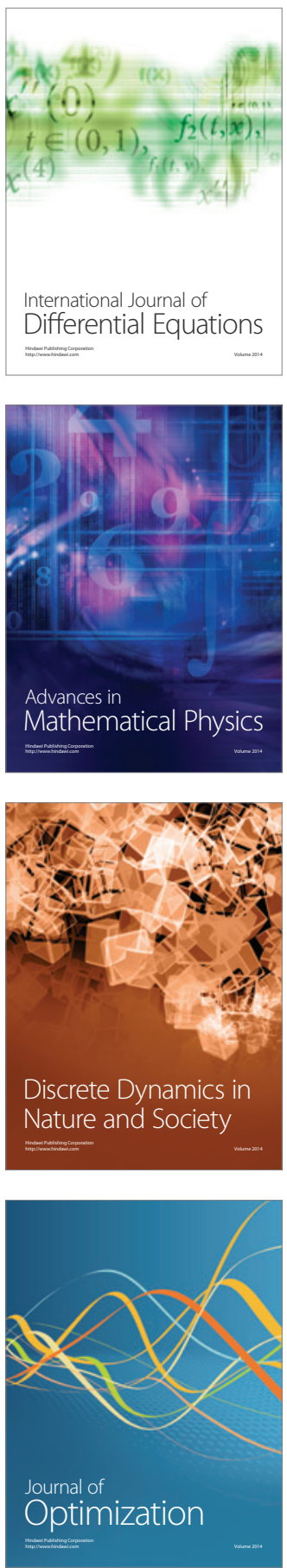